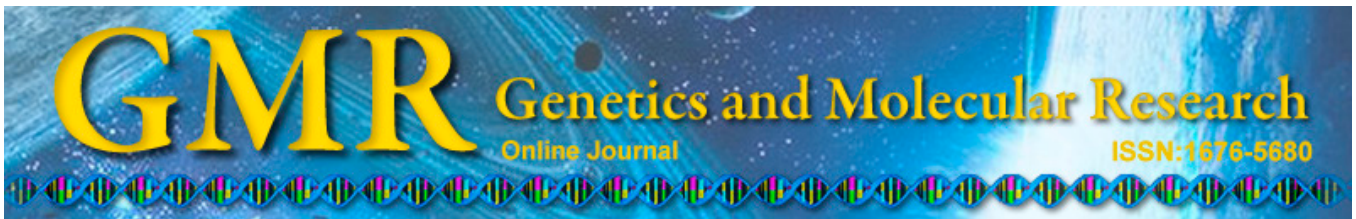

\title{
Development-related expression patterns of protein-coding and miRNA genes involved in porcine muscle growth
}

\author{
F.J. Wang, L. Jin, Y.Q. Guo, R. Liu, M.N. He, M.Z. Li and X.W. Li \\ Institute of Animal Genetics and Breeding, \\ College of Animal Science and Technology, Sichuan Agricultural University, \\ Ya'an, Sichuan, China \\ Corresponding author: X.W. Li \\ E-mail: xuewei.li@sicau.edu.cn
}

Genet. Mol. Res. 13 (4): 9921-9930 (2014)

Received January 9, 2014

Accepted August 19, 2014

Published November 27, 2014

DOI http://dx.doi.org/10.4238/2014.November.27.21

\begin{abstract}
Muscle growth and development is associated with remarkable changes in protein-coding and microRNA (miRNA) gene expression. To determine the expression patterns of genes and miRNAs related to muscle growth and development, we measured the expression levels of 25 protein-coding and 16 miRNA genes in skeletal and cardiac muscles throughout 5 developmental stages by quantitative reverse transcription-polymerase chain reaction. The Short Time-Series Expression Miner (STEM) software clustering results showed that growth-related genes were downregulated at all developmental stages in both the psoas major and longissimus dorsi muscles, indicating their involvement in early developmental stages. Furthermore, genes related to muscle atrophy, such as forkhead box 1 and muscle ring finger, showed unregulated expression with increasing age, suggesting a decrease in protein synthesis during the later stages of skeletal muscle development. We found that development of the cardiac muscle was a complex process in which growth-related genes were highly expressed
\end{abstract}


during embryonic development, but they did not show uniform postnatal expression patterns. Moreover, the expression level of miR-499, which enhances the expression of the $\beta$-myosin heavy chain, was significantly different in the psoas major and longissimus dorsi muscles, suggesting the involvement of miR-499 in the determination of skeletal muscle fiber types. We also performed correlation analyses of messenger RNA and miRNA expression. We found negative relationships between miR-486 and forkhead box 1, and miR-133a and serum response factor at all developmental stages, suggesting that forkhead box 1 and serum response factor are potential targets of miR-486 and miR-133a, respectively.

Key words: Muscle growth; Porcine; Expression pattern; qRT-PCR

\section{INTRODUCTION}

The muscle tissue is one of 4 fundamental types of tissues present in animals and mainly consists of skeletal muscle and cardiac muscle (CM). Both skeletal muscle and CM are striated because they contain sarcomeres that are packed into highly regular arrangements of bundles. Skeletal muscles, the heaviest tissues that account for $\sim 40 \%$ of total body mass (Pedersen and Febbraio, 2012), are the predominant sites of energy metabolism and the dominant organ system involved in locomotion. Conversely, CM is controlled by the autonomic nervous system. In animal growth and developmental processes, muscle mass is the most important determinant of the quality of carcass, which is directly related to efficient production. The gain of muscle mass is a complex process dependent on protein and muscle fiber turnover. Notably, the gain of skeletal muscle fibers mainly occurs during embryonic development, whereas hypertrophy of the skeletal muscle occurs after birth. CM shows sustained growth from the embryonic period to adulthood and then almost completely halts and maintains a relatively stable state thereafter. Many studies have revealed the important roles of protein-coding and microRNA (miRNA) genes in the regulation of muscle growth (Sandri, 2008; van Rooij et al., 2008). However, the changes in expression of these genes with increasing age and the interactions of miRNAs with protein-coding genes have not been fully defined in muscle development.

Pigs are commonly used as animal models in biomedical research because their physiology and anatomy show a considerable resemblance to those of humans (Garthoff et al., 2002). We explored the expression patterns of 25 protein-coding (Table 1) and 16 miRNA genes related to the growth and development of 3 porcine muscle tissues [i.e., CM, psoas major muscle (PMM), and longissimus dorsi muscle (LDM)] throughout 5 developmental stages [i.e., embryonic day (E) 90; postnatal days (P) 0, 30, and 180; and 7 years (Y) of age] to investigate variations in the expression levels of these genes with increasing age.

\section{MATERIAL AND METHODS}

\section{Sample collection and measurement}

The female Jinhua pigs across E90, P0, P30, P180, and 7Y were used in this study, and 3 individuals were randomly selected from each developmental stage. Animals were humanely 
sacrificed to ameliorate suffering. CM, PMM, and LDM were collected from each animal; the samples were frozen in liquid nitrogen immediately and stored at $-80^{\circ} \mathrm{C}$.

\begin{tabular}{|c|c|c|c|}
\hline Gene symbol & Forward & Reverse & Product length \\
\hline PAX3 & atcggctaatcctgacatgc & acggtgggaaacttttgatg & 140 \\
\hline MSTN & aacagcgagcaaaaggaaaa & atcaatcagttcceggagtg & 201 \\
\hline MTOR & ctcgtcactcctctcaac & ccgettccttatggttct & 99 \\
\hline $\mathrm{NF} \kappa \mathrm{B}$ & gcaccctgatcttgettatt & gaggtccatctcettcgtct & 115 \\
\hline SMAD2 & cttctggctcagtccgtta & ctgtctgcettcggtattct & 115 \\
\hline FOXO1 & getttacaagtgcetctgcc & tctccatccatgaggtcgtt & 197 \\
\hline MRF4 & cttgagggtgcggatttcetg & ctgaagactgctggaggetg & 132 \\
\hline IGF1 & ctggtggacgctcttcagtt & acatctccagcetcctcaga & 151 \\
\hline MURF & gtgacaaaggcaagaccc & acacggcaagatgaccacc & 195 \\
\hline MYF4 & caaccaggaggagcgagacctccg & aggcgctgtgggatatgcattcact & 86 \\
\hline MYOD & gcactacagcggtgactcag & cacgatgctggacagacagt & 196 \\
\hline SMAD3 & ggagaagtggtgcgagaagg & cacaggcggcagtagatgac & 195 \\
\hline S6K1 & gaggacatggcaggagtgtt & cctttaccaagtacccgaag & 236 \\
\hline MyHC & ctggatgccagtgagcgtgtc & gcgttgcgagcttcctgaata & 134 \\
\hline PDK1 & aatcaccaggacagccaata & ctcggtcactcatcttcaca & 186 \\
\hline Follistatin & ggcctatgagggaaagtgta & ctcggtgtcttctgaaatgg & 274 \\
\hline MYF5 & tgccagttctcgecttctga & tttcctcttgcacgctttgc & 216 \\
\hline IGF1R & tggatgccgtgtccaat & gtgtcgttgtcgggtgc & 256 \\
\hline SRF & gactggcaaggcactgattc & tgctgtctggattgtggagg & 220 \\
\hline PI3K & tgaaagtagattggctggac & cactatctcaaagcccgtta & 197 \\
\hline GSK3 & tacgggacccaaatgtcaaa & acgcagaagcggtgttattg & 216 \\
\hline MEF2A & gaccctgatacttcctatgtgc & tgaactccetgggttagtgtag & 189 \\
\hline AKT1 & atcgtgtggcaggatgtgta & ctggccgagtaggagaactg & 200 \\
\hline MAPK14 & gattctccgaggtctcaa & gccacatagcctgtcatt & 153 \\
\hline IRS1 & ctgattggcatctaccgc & gcctccaggattgtctcat & 233 \\
\hline
\end{tabular}

\section{RNA extraction}

Total RNA was isolated from the muscle tissues using RNAisoPlus (TaKaRa, Dalian, China), purified using RNeasy columns (Qiagen, Duesseldorf, Germany), and RNA concentrations were measured by the NanoDrop 2000 (Thermo Scientific, USA) according to the manufacturer protocol.

\section{Quantitative real-time polymerase chain reaction (qRT-PCR)}

Total RNA was reverse transcripted following manufacturer recommendations for: 1) mRNA transcribed into complementary DNA (cDNA) using the oligo(dT) and random 6-mer primers provided in the PrimeScript RT MasterMix kit (TaKaRa); and 2) miRNA using the oligo(dT) and poly(A) polymerase provided in the PrimeScript miRNA RT-PCR Kit (TaKaRa). qRT-PCR was performed using the SYBR Premix Ex Taq kit (TaKaRa) on a CFX96 real-time PCR detection system (Bio-Rad, Richmond, Canada).

\section{Statistical data and biological analysis}

According to Figure S1, all mRNA expression levels were normalized by the 3 housekeeping genes with greater stability (i.e., PPIA, RPL4, and YWHAZ), while miRNA 
expression levels were normalized by 3 other housekeeping genes (i.e., $18 \mathrm{~S}, 5 \mathrm{~S}$, and $\mathrm{U6}$ ). All experiments contained a negative control, and each qRT-PCR was performed in triplicate.

\section{RESULTS AND DISCUSSION}

\section{Protein-coding gene expression patterns at various developmental stages}

Growth of muscle mass is a time-dependent process regulated by protein-coding genes and other factors. To investigate the expression levels of these genes, we used the Short Time-Series Expression Miner (STEM) software for protein coding and miRNA gene clustering.

Formation and function of the heart occur at early stages during embryonic development, which accounts for all subsequent events in the life of an organism (Olson, 2004). Although functional development of the heart is completed during the embryonic period, it grows actively after birth into adulthood (Manabe et al., 2002; McMullen et al., 2004). Growth factors cooperate to regulate the early growth of CM. These factors exhibited increasing expression levels from the prenatal to early postnatal stages but decreased at later stages (Figure 1a; Table S1) and showed extremely similar expression patterns $(\mathrm{P}<$ $0.001)$. Incidentally, we found that the muscle ring finger $(M U R F)$, follistatin, and mitogen-activated protein kinase $(M A P K)$ genes were significantly clustered into one group ( $\mathrm{P}$ $=0.040$ ), indicating a unique expression pattern throughout the 5 developmental stages. This expression patterns in CM showed strong upregulation from E90 to P0, downregulation from P0 to P30, upregulation from P30 to P180, and downregulation at 7Y. During $\mathrm{CM}$ development, the follistatin gene may participate in positive regulation of muscle mass. Postnatally, the gain of muscle slows and is regulated by numerous factors, which is a complicated regulatory process.

The gain or loss of skeletal muscle mass involves a delicate balance between the production of new myofibrils and degradation of existing proteins (Sandri, 2008), which is directly regulated by protein-coding genes. In both the LDM and PMM, muscle regulatory factor 4 (MRF4), forkhead box 1 (FOXO1), and MURF exhibited slow rises in expression levels throughout the 5 stages (Figure $1 \mathrm{~b}$ and c; Table S1). MRF4 is the only gene of the MYOD family that is expressed in embryonic myotomes to promote muscle mass and continues to increase postnatally (Hinterberger et al., 1991). Moreover, FOXO1 can reduce muscle mass and fiber atrophy (Xu et al., 2012), and MURF ubiquitinates and degrades myosin heavy chains (Clarke et al., 2007); all of these changes negatively regulate muscle mass. Skeletal muscle shows remarkable changes in metabolism with increasing age, which is reflected by the distribution and size of the muscle fibers and a general deceleration in muscle mass gain (Carmeli and Reznick, 1994). We found that the expression patterns of $M U R F$ and $F O X O 1$ were upregulated with age, suggesting a decrease in the deposition of protein with increasing age. $\beta$-myosin heavy chain $(\beta-M Y H C)$, the determinant gene of skeletal muscle isoforms (fast or slow) (Agbulut et al., 2003), showed different expression patterns in LDM (fast muscle) and PMM (slow muscle), which is consistent with previous studies (Figure 2). 

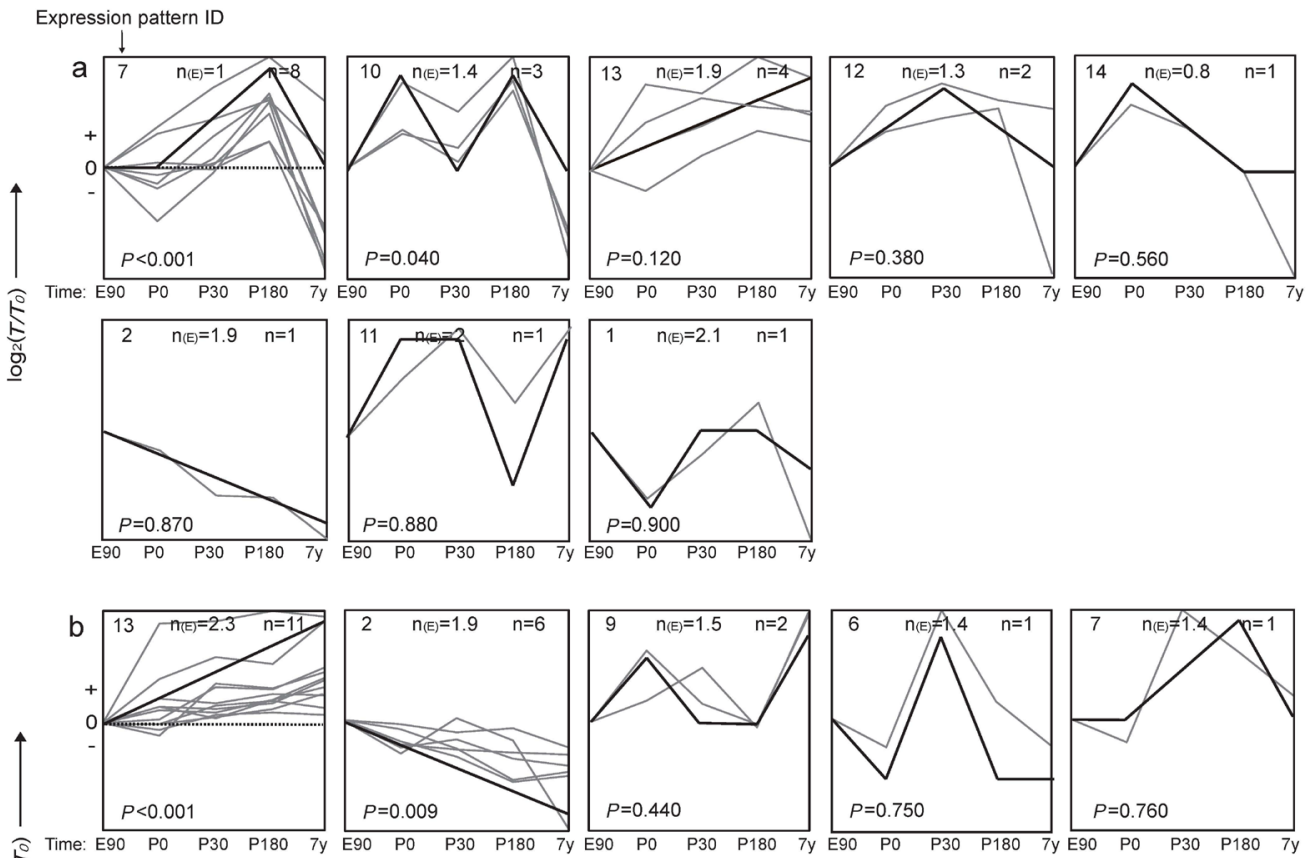

ह
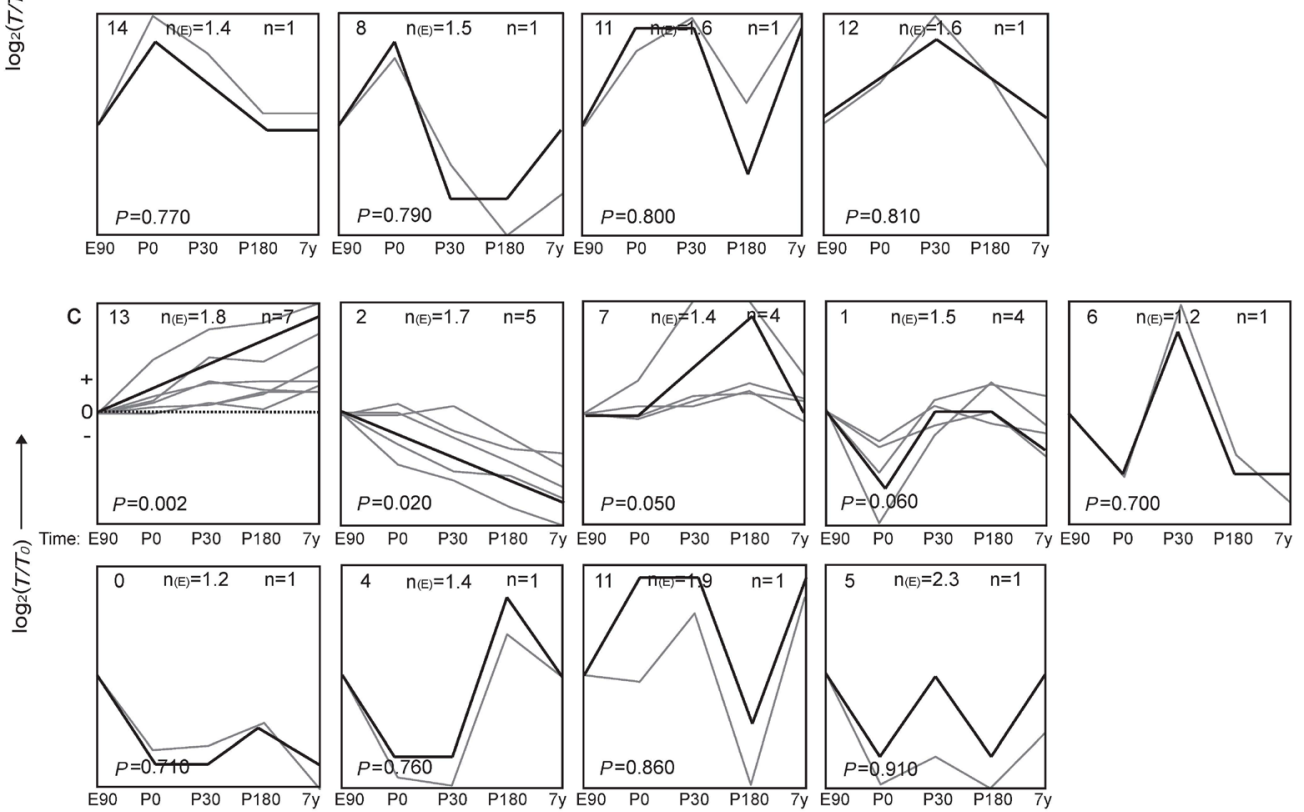

Figure 1. Short Time-Series Expression Miner (STEM) clustering profiles for protein-coding genes in: (a) cardiac muscle (CM); (b) longissimus dorsi muscle (LDM); and (c) psoas major muscle (PMM). The dashed line indicates no change in expression among the different stages. The number at the top left of each square is the expression pattern ID. The black, bold-type lines in the squares represent the trend lines of the expression patterns, and the gray lines represent the variation in expression of the protein-coding genes from embryonic day 90 (E90) to 7 years (7Y). 


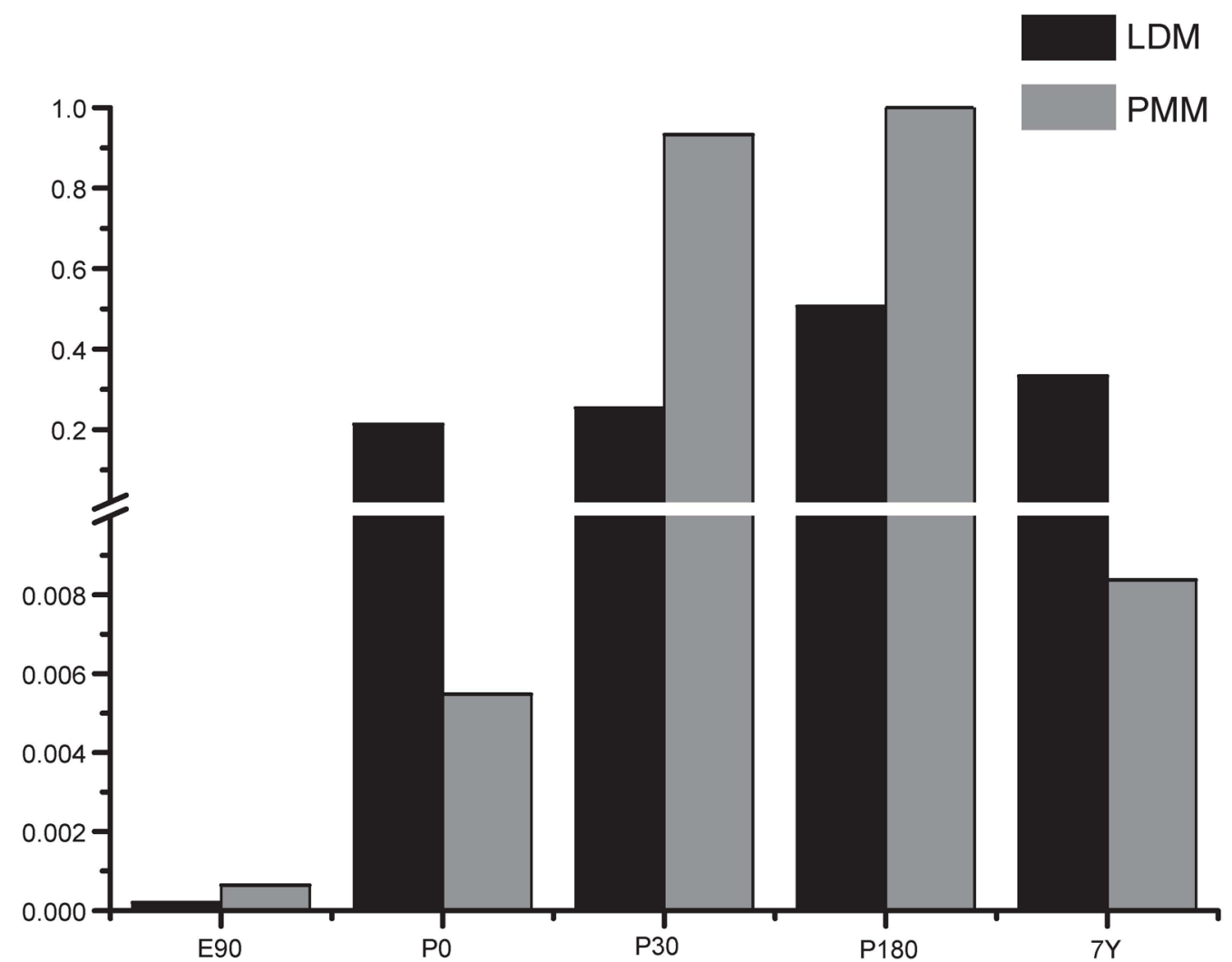

Figure 2. $\beta$-myosin heavy chain $(\beta$-MYHC) expression pattern in longissimus dorsi muscle (LDM) and psoas major muscle (PMM).

\section{miRNA expression patterns at various developmental stages}

miRNAs play important roles in the regulatory networks of protein-coding genes related to muscle development. They participate in diverse aspects of muscle function, including myoblast proliferation, differentiation, and muscle atrophy (van Rooij et al., 2008; TownleyTilson et al., 2010; Xu et al., 2012).

In CM, among the 16 miRNAs, 7 miRNAs showed a dramatic decrease from E90 to 7Y (Figure 3; Table S2). miR-1, miR-206, miR-486, miR-221, miR-195, miR-320, and miR24 , which play important roles in muscle myogenesis, differentiation, or proliferation, showed higher expression levels during the early developmental stages when compared to those during later developmental stages (Callis et al., 2007; Carè et al., 2007; Townley-Tilson et al., 2010). For example, miR-486, which directly suppresses $\operatorname{Pax} 7$ expression, modulates myoblast development from the proliferation stage to the subsequent differentiation phase during embryonic development (Dey et al., 2011). Furthermore, it has been shown that this process is completed during the embryonic period; then, the expression level of miR-486 decreases postnatally (Xi et al., 2007).

Notably, in skeletal muscle, we found that miR-1 and miR-499 showed the highest 
expression levels at E90 in both the LDM and PMM (Figure 3). According to previous reports, miR-1 plays an important role in skeletal muscle development of Drosophila and Xenopus laevis embryos (Chen et al., 2005; Nguyen and Frasch, 2006) and is highly expressed during muscle cell differentiation. Our results suggest a role of miRNA-1 in the regulation of muscle cell differentiation during the embryonic period of pigs. Moreover, miR-499 is expressed in skeletal muscle during mouse embryogenesis (van Rooij et al., 2009). In this study, we identified a high expression level of miR-499 during the embryonic period, but its precise function in early skeletal muscle development is currently unknown. miR-499 is expressed in skeletal muscle and controls the skeletal muscle fiber type after birth, which has been validated in transgenic mice (van Rooij et al., 2009). We found that miR-499 had a higher $(\mathrm{P}<0.001)$ expression level in PMM than that in LDM, suggesting its role in the formation of type I myofibers in pigs.
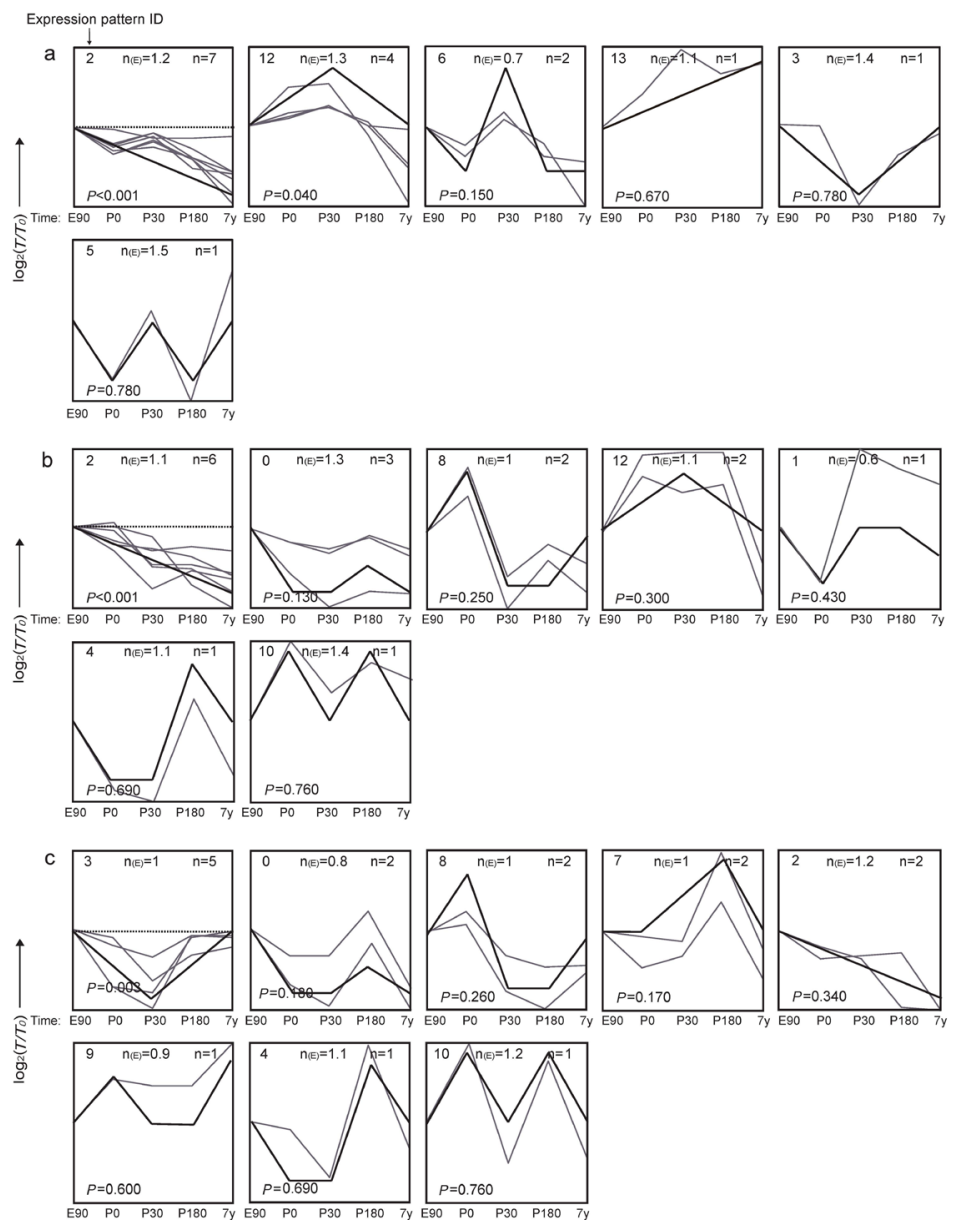

Figure 3. STEM clustering profiles for microRNAs (miRNAs) in CM (a), LDM (b) and PMM (c) The dashed line indicates no change in expression among the different stages. The number in the top left of each square is the expression pattern ID. The black, bold-type lines in the squares indicate the trend lines of the expression patterns, and the gray lines represent the variation in expression of miRNAs from E90 to 7Y. 


\section{Relationship between protein-coding genes and miRNAs at various developmental stages}

miRNAs negatively regulate gene expression by promoting target mRNA degradation and inhibiting mRNA translation (van Rooij et al., 2008). However, these interactions have generally been elucidated in transgenic mouse models or at the cellular level. To determine whether these interactions were present during various developmental stages, we constructed a correlative network of genes and miRNAs during muscle growth and development (Figure S2).

We found that in insulin-like growth factor 1 (IGF1)-miR-1, IGF1-miR-320, serum response factor (SRF)-miR-133a, and FOXO1-miR-486 pairs (Figure 4), the expression level of miRNA and its target gene showed significant negative correlations. IGF1 plays an important role in muscle cell proliferation and myogenic differentiation, and its expression level decreases with increasing age. Previous studies have shown that $I G F 1$ is a target of miR-1 and miR-320 (Musarò et al., 2001; Wang et al., 2009). SRF inhibits muscle cell proliferation and differentiation in vitro and in vivo (Wang et al., 2002; Li et al., 2005), and its expression level increases after birth and subsequently stabilizes in adulthood. miR-133 is mainly expressed during embryogenesis and promotes muscle cell proliferation by directly repressing the expression of $S R F$. FOXO1 is usually associated with muscle waste disease or aging under normal conditions, and its expression level increases during the late developmental stages. In addition, FOXO1 is a target of miR-486 (Small et al., 2010), and they jointly participate in protein degradation. The decreasing level of miR-486 with age suggests an increasing expression level of FOXO1, which may promote muscle atrophy during the later stages of pig muscle development.

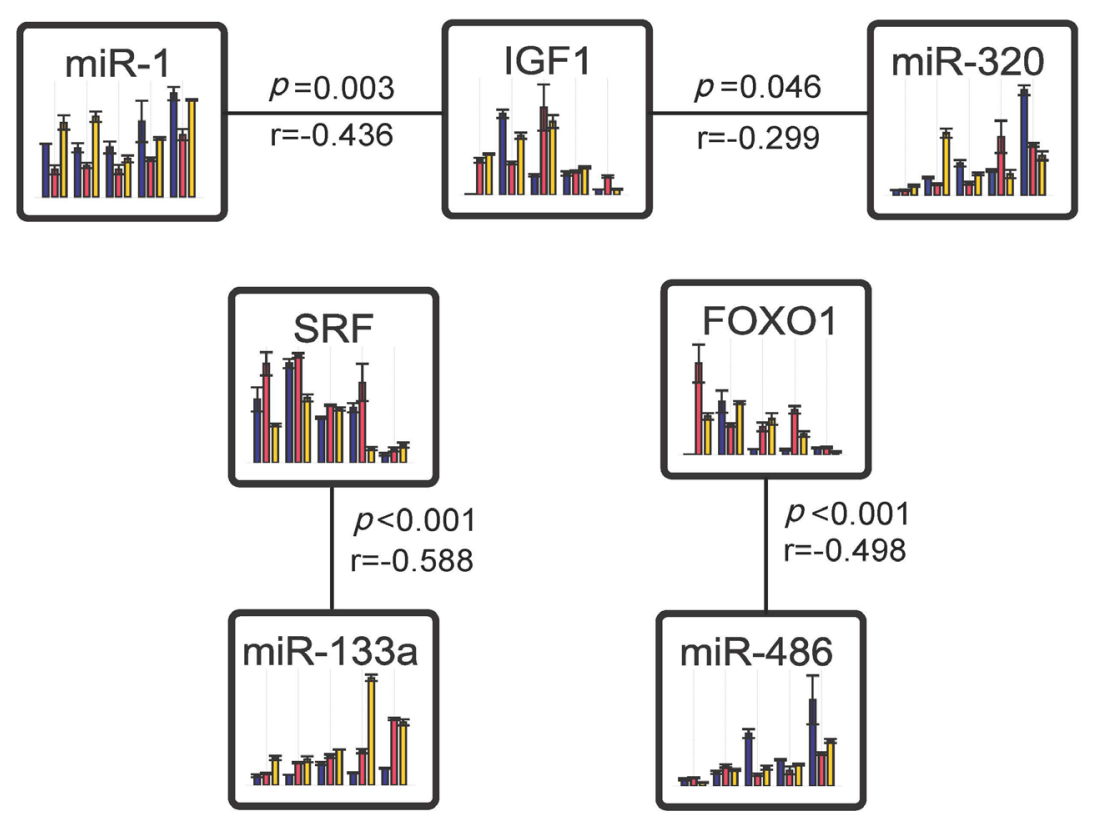

Figure 4. Expression patterns of protein-coding gene-miRNA pairs. The squares represent the expression patterns of miRNA or protein-coding gene in CM (blue bars), LDM (red bars), and PMM (yellow bars). From left to right, E90, P0, P30, P180, and 7Y are represented, respectively. 
In summary, we investigated the expression changes of porcine protein-coding and miRNA genes related to muscle development by qRT-PCR. Combined with STEM clustering, we found that growth-related genes are highly expressed during the embryonic period in $\mathrm{CM}$, but do not show uniform expression patterns after birth. Moreover, most genes related to muscle hypertrophy are downregulated with increasing age in skeletal muscle, while the genes related to muscle atrophy are upregulated. In addition, some mRNAs and miRNAs exhibited significant negative correlations throughout all developmental stages. At present, we have constructed only a crude genetic blueprint of muscle development, with a multitude of details that have yet to be clarified. Further study utilizing more accurate methods, such as RNA-seq or small RNA-seq, will be needed to delineate the complicated mechanisms of gene regulation during muscle development.

\section{ACKNOWLEDGMENTS}

Research supported by grants from the National Special Foundation for Transgenic Species of China (\#2014ZX0800950B and \#2011ZX08006-003), the Fund for Distinguished Young Scientists of Sichuan Province (\#2013JQ0013), the Specialized Research Fund of Ministry of Agriculture of China (\#NYCYTX-009), and the Project of Provincial Twelfth Five Years' Animal Breeding of Sichuan Province (\#2011YZGG15).

\section{Supplementary material}

\section{REFERENCES}

Agbulut O, Noirez P, Beaumont F and Butler-Brown G (2003). Myosin heavy chain isoforms in postnatal muscle development of mice. Biol. Cell 95: 399-406.

Callis TE, Chen JF and Wang DZ (2007). MicroRNAs in skeletal and cardiac muscle development. DNA Cell Biol. 26: 219-225.

Carè A, Catalucci D, Felicetti F, Bonci D, et al. (2007). MicroRNA-133 controls cardiac hypertrophy. Nat. Med. 13: 613618.

Carmeli E and Reznick AZ (1994). The physiology and biochemistry of skeletal muscle atrophy as a function of age. $P$. Soc. Exp. Biol. Med. 206: 103-113.

Chen JF, Mandel EM, Thomson JM, Wu Q, et al. (2005). The role of microRNA-1 and microRNA-133 in skeletal muscle proliferation and differentiation. Nat. Genet. 38: 228-233.

Clarke BA, Drujan D, Willis MS, Murphy LO, et al. (2007). The E3 Ligase MuRF1 degrades myosin heavy chain protein in dexamethasone-treated skeletal muscle. Cell Metab. 6: 376-385.

Dey BK, Gagan J and Dutta A (2011). miR-206 and -486 induce myoblast differentiation by downregulating Pax7. Mol. Cell. Biol. 31: 203-214.

Garthoff LH, Henderson GR, Sager AO, Sobotka TJ, et al. (2002). The Autosow raised miniature swine as a model for assessing the effects of dietary soy trypsin inhibitor. Food Chem. Toxicol. 40: 487-500.

Hinterberger TJ, Sassoon DA, Rhodes SJ and Konieczny SF (1991). Expression of the muscle regulatory factor MRF4 during somite and skeletal myofiber development. Dev. Biol. 147: 144-156.

Li S, Czubryt MP, McAnally J, Bassel-Duby R, et al. (2005). Requirement for serum response factor for skeletal muscle growth and maturation revealed by tissue-specific gene deletion in mice. Proc. Natl. Acad. Sci. U S A 102: 10821087.

Manabe I, Shindo T and Nagai R (2002). Gene expression in fibroblasts and fibrosis involvement in cardiac hypertrophy. Circ. Res. 91: 1103-1113.

McMullen JR, Shioi T, Huang WY, Zhang L, et al. (2004). The insulin-like growth factor 1 receptor induces physiological heart growth via the phosphoinositide 3-kinase (p110alpha) pathway. J. Biol.Chem. 279: 4782-4793.

Musarò A, McCullagh K, Paul A, Houghton L, et al. (2001). Localized Igf-1 transgene expression sustains hypertrophy 
and regeneration in senescent skeletal muscle. Nat. Genet. 27: 195-200.

Nguyen HT and Frasch M (2006). MicroRNAs in muscle differentiation: lessons from Drosophila and beyond. Curr. Opin. Genet. Dev. 16: 533-539.

Olson EN (2004). A decade of discoveries in cardiac biology. Nat. Med. 10: 467-474.

Pedersen BK and Febbraio MA (2012). Muscles, exercise and obesity: skeletal muscle as a secretory organ. Nat. Rev. Endocrinol. 8: 457-465.

Sandri M (2008). Signaling in muscle atrophy and hypertrophy. Physiology 23: 160-170.

Small EM, O'Rourke JR, Moresi V, Sutherland LB, et al. (2010). Regulation of PI3-kinase/Akt signaling by muscleenriched microRNA-486. P. Natl. Acad. Sci. U S A 107: 4218-4223.

Townley-Tilson WH, Callis TE and Wang D (2010). MicroRNAs 1, 133, and 206: critical factors of skeletal and cardiac muscle development, function, and disease. Int. J. Biochem. Cell Biol. 42: 1252-1255.

van Rooij E, Liu N and Olson EN (2008). MicroRNAs flex their muscles. Trends Genet. 24: 159-166.

van Rooij E, Quiat D, Johnson BA, Sutherland LB, et al. (2009). A family of microRNAs encoded by myosin genes governs myosin expression and muscle performance. Dev. Cell 17: 662-673.

Wang D, Passier R, Liu ZP, Shin CH, et al. (2002). Regulation of cardiac growth and development by SRF and its cofactors. Cold Spring Harb. Symp. Quant. Biol. 67: 97-105.

Wang XH, Qian RZ, Zhang W, Chen SF, et al. (2009). MicroRNA-320 expression in myocardial microvascular endothelial cells and its relationship with insulin-like growth factor-1 in type 2 diabetic rats. Clin. Exp. Pharmacol. Physiol. 36: 181-188.

Xi G, Hathaway MR, Dayton WR and White ME (2007). Growth factor messenger ribonucleic acid expression during differentiation of porcine embryonic myogenic cells. J. Anim. Sci. 85: 143-150.

Xu J, Li R, Workeneh B, Dong YL, et al. (2012). Transcription factor FoxO1, the dominant mediator of muscle wasting in chronic kidney disease, is inhibited by microRNA-486. Kidney Int. 82: 401-411. 